\title{
Intramural gastric air - gastric pneumatosis or emphysematous gastritis?
}

Werner Harmse, MB ChB
Vicci Smith, MB ChB, DA (SA)
Aisne Stoker, MB ChB, DMRD, FRCR
Department of Radiology, Pietermaritzburg Hospital Complex,
Nelson R Mandela School of Medicine, University of KwaZulu-Natal

\section{Abstract}

Gas in the wall of the stomach is a rare condition and may be caused by gastric pneumatosis (also known as gastric emphysema) or emphysematous gastritis. We present the case of a 27 -year-old white female patient with a history of thoracic and abdominal surgery who presented with intractable vomiting, clinically suspected to be as a result of gastric outlet obstruction. Intramural gastric air was subsequently demonstrated on plain abdominal X-rays, fluoroscopy (water-soluble contrast meal), and on an abdominal CT scan.

\section{Introduction}

Gastric pneumatosis (also known as gastric emphysema) and emphysematous gastritis are terms describing air in the wall of the stomach.

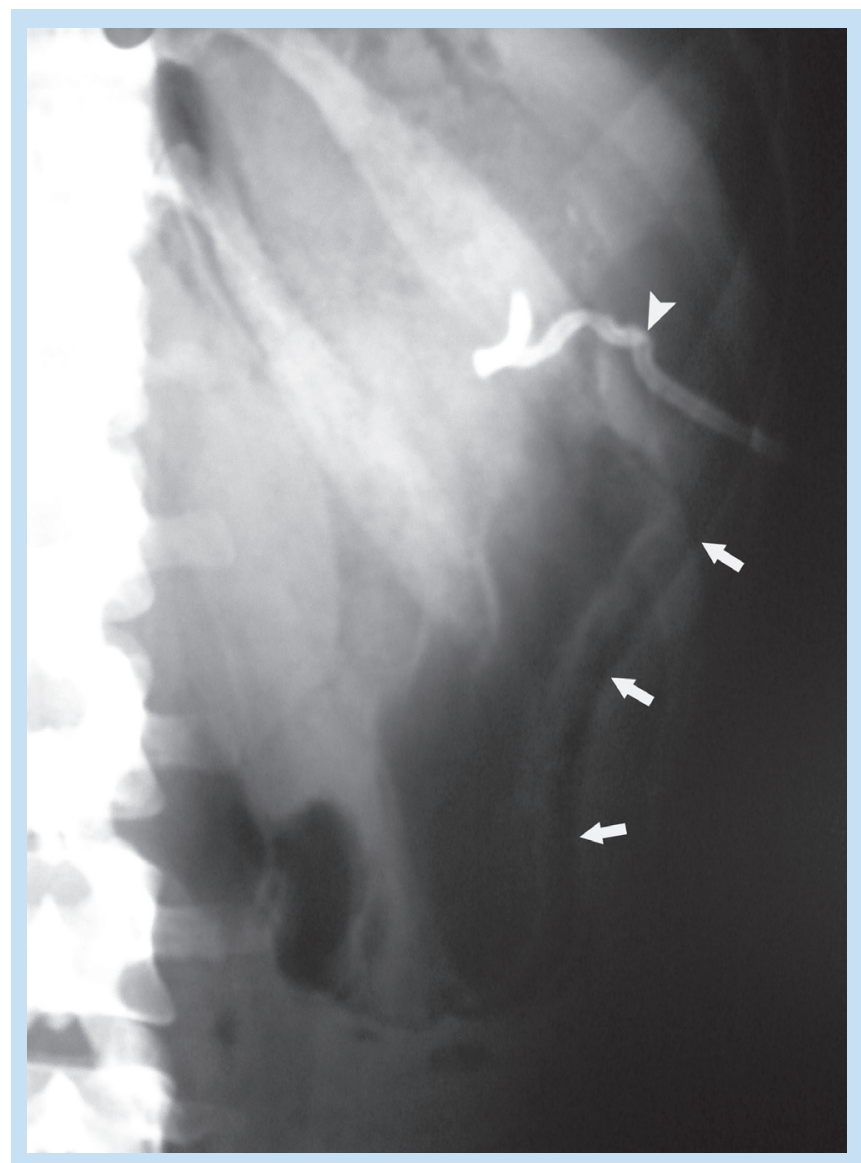

Fig. 1. Plain film demonstrating linear intramural gastric air (arrows) and the left anterior subphrenic percutaneous drain (arrowhead).

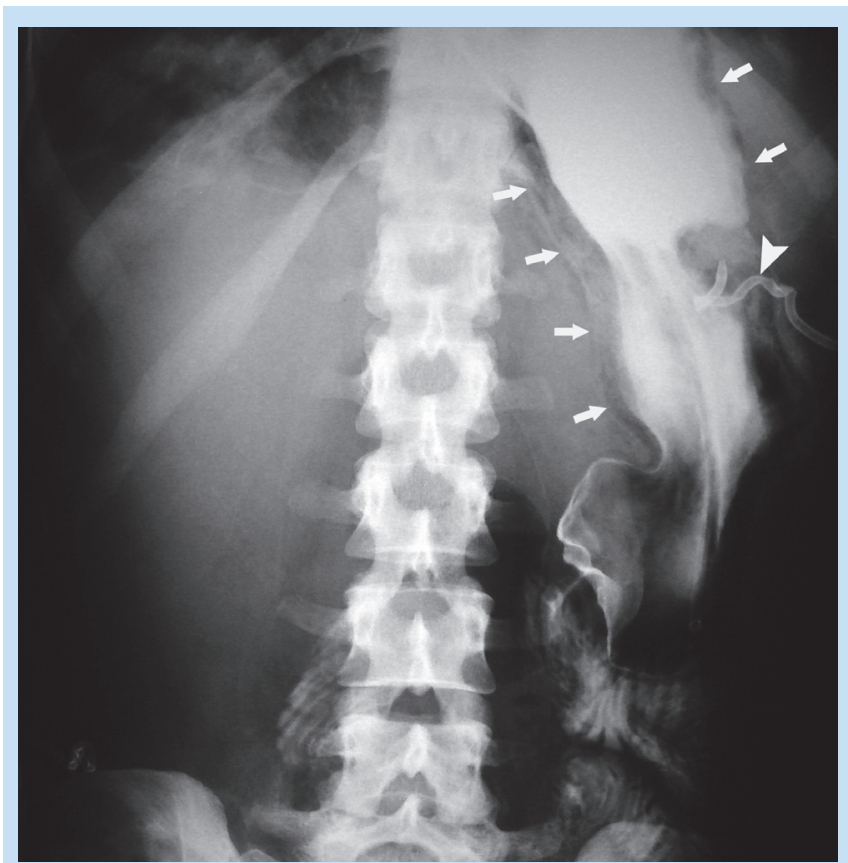

Fig. 2. Water-soluble contrast meal demonstrating intramural gastric air (arrows) and the left anterior subphrenic percutaneous drain (arrowhead).

Intramural gastric air is a rare clinical condition. It was first described by Chamberlain in $1947 .{ }^{1}$ Cases in the literature have described radiological, endoscopic, histological and trans-abdominal ultrasound findings in adult and paediatric populations.

\section{Case report}

A female patient, aged 27 years, presented with intractable vomiting, clinically diagnosed as gastric outlet obstruction. At this point the patient was afebrile and abdominal pain was not present. Plain abdominal radiographs (Fig. 1) and a water-soluble contrast meal (Fig. 2) demonstrated linear streaks of gas in the gastric wall. There were no gas loculi present and there was no thickening of the gastric rugae. A CT scan of the abdomen confirmed the presence of intramural gastric air (Fig. 3).

Four months prior to this episode, the patient delivered a healthy full-term infant via a normal vaginal delivery. There had been no prenatal complications. Ten days following delivery she presented with dyspnoea. A chest X-ray demonstrated a large mass adjacent to the right hemi-diaphragm. A thoracotomy was performed, the mass was excised and the differential diagnosis on histology was a pleomorphic rhabdomyosarcoma or a liposarcoma.

One month following the thoracotomy, the patient developed a low small-bowel obstruction. Surgery was undertaken and perforations of the ileum and urinary bladder were found. An ileostomy was consequently performed. During her hospital stay, two further laparotomies and abdominal wash-outs were performed, as well as an ultrasoundguided percutaneous drainage of a left anterior sub-phrenic abscess. 


\section{CASE REPORT}

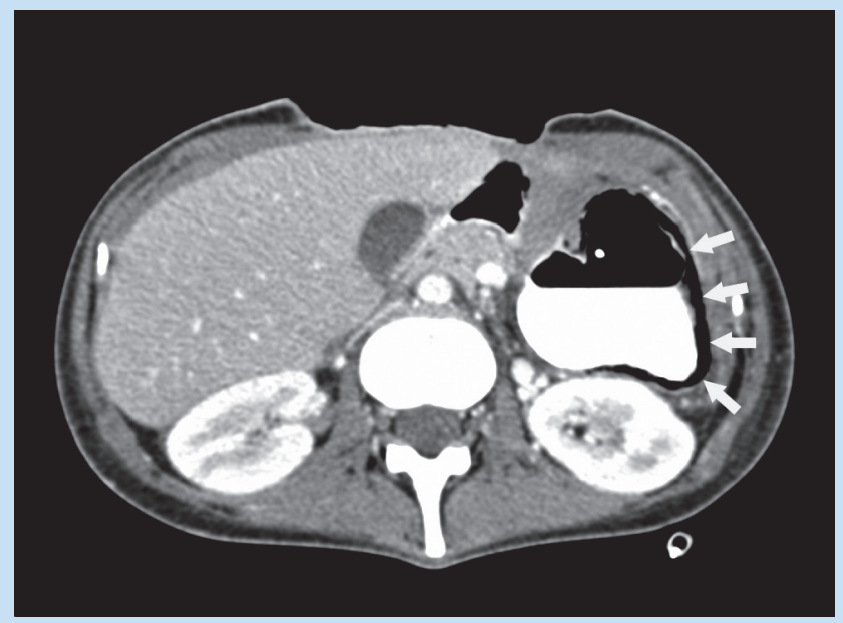

Fig. 3. CT of the abdomen demonstrating linear intramural gastric air (arrows).

Two weeks after the percutaneous drainage she developed intractable vomiting and the water-soluble contrast meal was performed, demonstrating the intramural gastric air.

The patient was treated empirically with broad-spectrum IV antibiotics and total parenteral nutrition (TPN) for 7 days. At that stage she was able to tolerate food, and subsequently recovered and was discharged 3 weeks later.

\section{Discussion}

Gas in the wall of the stomach is a rare radiological finding, which can be caused by gastric pneumatosis or emphysematous gastritis. These two conditions can be distinguished on the bases of pathogenesis, clinical picture and radiological appearance, although they may mimic each other.

Gastric pneumatosis or gastric emphysema describes the presence of gas within the stomach wall. This is caused by a disruption in gastric mucosa leading to the dissection of air into the wall. There are various causes for this phenomenon: $(i)$ conditions causing raised intragastric pressure, i.e. gastric outlet obstruction; (ii) post-gastroscopy (air insufflation and other instrumentation may cause mucosal damage); (iii) severe vomiting, which causes mucosal damage; (iv) dissection of air from the mediastinum (e.g. ruptured bullae or pneumothorax). ${ }^{2,3}$ These patients are usually asymptomatic or complain of mild dysphagia and/or epigastric discomfort. Plain radiographs characteristically demonstrate a linear lucency conforming to the contour of a thin-walled, often distended, stomach, and enveloping any intraluminal gas and fluid content. ${ }^{2,3}$ The clinical course is usually benign, with spontaneous resolution after the cause is removed. In these cases, gas is not produced within the stomach wall but enters it from elsewhere. ${ }^{2}$ This should be the main criterion in making the diagnosis of gastric pneumatosis/emphysema.
In comparison, emphysematous gastritis occurs when there is diffuse infiltration of the stomach wall by pathogenic gas-forming bacteria. Thus the gas is formed within the stomach wall. In these cases, an earlier gastric mucosal injury allows gas-forming organisms to gain access to deeper tissue layers. Causes of this condition include: (i) ingestion of toxic or corrosive substances; (ii) alcohol ingestion; (iii) trauma; (iv) gastric infarction; $(v)$ ulcer disease; $(v i)$ necrotising enterocolitis. ${ }^{5}$ The most common organisms involved include haemolytic streptococci, Clostridia welchi, Escherichia coli and Staphylococcus aureus. These patients present with severe, acute epigastric pain, fever, shock, toxaemia, nausea, often haematemesis and a leucocytosis. Radiologically, the stomach is often contracted with numerous frothy or mottled radiolucencies (gas bubbles) in the gastric wall. ${ }^{2,4}$ These lucencies silhouette the stomach outline and remain constant with positional change. Other features may include gas in the portal venous system and thickened rugal folds. Contrast studies may demonstrate a cobblestone appearance of the mucosa due to submucosal blebs, and occasionally intramural penetration of contrast may be seen. Abdominal CT may further distinguish this from gastric pneumatosis/emphysema by detecting small amounts of intramural gas as well as gastric wall thickening. Emphysematous gastritis has a uniformly poor prognosis with a mortality of $60-80 \%{ }^{2,5}$ Treatment is usually conservative with broad-spectrum antibiotics, intravenous fluids and nutritional support being the mainstay. If the patient survives, cicatricial stenosis may occur, which requires surgery.

In the case we present, the patient was not toxically ill at the time of demonstration of intramural gastric air. The radiographs showed a streaky intramural gas pattern. We therefore diagnosed this case as gastric pneumatosis/emphysema and hypothesised that her condition was as a result of her previous surgical interventions. Her uneventful recovery and subsequent discharge from hospital further substantiates our diagnosis.

\section{Conclusion}

Intramural gastric air is a rare but important sign to recognise and diagnose as it may represent either one of two conditions, which differ vastly in terms of clinical and radiographic presentation, management plan and prognosis. Patients with gastric pneumatosis are clinically asymptomatic, and a streaky or linear air pattern is demonstrated radiographically. The prognosis is excellent. In contrast, patients with emphysematous gastritis are usually severely ill and the gas pattern has a mottled or bubbly radiographical appearance. The prognosis is uniformly poor.

1. Chamberlain DT. Pneumoperitoneum following gastroscopy apparently without perforation: report of a case. New Engl J Medicine 1947; 237: 843-845.

2. Fidvi SA, Klein SA. Clinical Quiz. Appl Radiol 2002; 31(3): 33-36.

3. Soon M, Yen H, Soon A, Lin O. Endoscopic ultrasonographic appearance of gastric emphysema. World J Gastroenterol 2005; 11(11): 1719-1721.

4. Tuck JS, Boobis LH. Case report: interstitial emphysema of the stomach due to perforated appendicitis. Clin Radiol 1987; 38: 315-317.

5. Herring W. Gastric emphysema. http://www.learningradiology.com/notes/ginotes/gastricemphysemapage

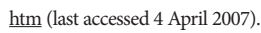

